\title{
A 3D endoscopic transtubular transcallosal approach to the third ventricle
}

\author{
Alireza Shoakazemi, MD, FRCS, ${ }^{1,2}$ Alexander I. Evins, MD, ${ }^{1}$ Justin C. Burrell, MS, ${ }^{1}$ \\ Philip E. Stieg, PhD, MD, ${ }^{1}$ and Antonio Bernardo, MD'1 \\ 1Department of Neurological Surgery, Weill Cornell Medical College, NewYork-Presbyterian Hospital, New York, New York; and \\ 2Department of Neurosurgery, Regional Neuroscience Unit, Royal Victoria Hospital, Belfast, United Kingdom
}

\begin{abstract}
OBJECT Surgical approaches to deep-seated brain pathologies, specifically lesions of the third ventricle, have always been a challenge for neurosurgeons. In certain cases, the transcallosal approach remains the most suitable option for targeting lesions of the third ventricle, although retraction of the fornices and wall of the third ventricle have been associated with neuropsychological and hypothalamic deficits. The authors investigated the feasibility of an interhemispheric 3D endoscopic transcallosal approach through a minimally invasive tubular retractor system for the management of third ventricular lesions.
\end{abstract}

METHODS Three-dimensional endoscopic transtubular transcallosal approaches were performed on 5 preserved cadaveric heads (10 sides). A parasagittal bur hole was placed using neuronavigation, and a tubular retractor was inserted under direct endoscopic visualization. Following observation of the vascular structures, fenestration of the corpus callosum was performed and the retractor was advanced through the opening. Transforaminal, interforniceal, and transchoroidal modifications were all performed and evaluated by 3 surgeons.

RESULTS This approach provided enhanced visualization of the third ventricle and more stable retraction of corpus callosum and fornices. Bayonetted instruments were used through the retractor without difficulty, and the retractor applied rigid, constant, and equally distributed pressure on the corpus callosum.

CONCLUSIONS A transtubular approach to the third ventricle is feasible and facilitates blunt dissection of the corpus callosum that may minimize retraction injury. This technique also provides an added degree of safety by limiting the free range of instrumental movement. The combination of 3D endoscopic visualization with a clear plastic retractor facilitates safe and direct monitoring of the surgical corridor.

http://thejns.org/doi/abs/10.3171/2014.11.JNS14341

KEY WORDS 3D endoscope; third ventricle; transcallosal; tubular retractor; transtubular; minimally invasive; surgical technique

0 INCE the introduction of retractors into neurosurgical practice by Greenberg in 1981, and subsequently by Rosenørn and Diemer in 1985 and Yokoh et al. in 1987, their clinical use has been subject to numerous modifications. ${ }^{13,14,31,39}$ While some have advocated for retractorless microsurgical techniques, increasing use of new surgical planning technologies has helped locate surgical routes that minimize brain retraction and resultant neural damage, and improve surgical outcomes. ${ }^{21}$ Stereo- tactic cylindrical retractors were first introduced by Kelly et al. in 1988 for the excision of deep intraparenchymal lesions ${ }^{20}$ Since then, tubular retractor design and application have been greatly improved and a number of different types of these systems have been introduced.

The endoscopic transcortical, interhemispheric transcallosal, and microsurgical transcortical approaches are commonly used routes to access lesions of the third ventricle. In specific cases, depending on the anatomy of the lesion and 
other clinical factors, the transcallosal approach may provide an optimal surgical corridor to the third ventricle. ${ }^{6,8,33}$ However, neuropsychological deterioration, memory deficit, and perioperative hypothalamic complications due to injury of the fornices, mammillary bodies, and/or hypothalamus have been reported to occur with this microsurgical approach..$^{15,25,29}$ Endoscope-assisted interhemispheric approaches for corpus callosotomy have been proposed for use in epilepsy surgery, but endoscopic transtubular approaches to the third ventricle through the transcallosal corridor have yet to be described. ${ }^{2,23,34} \mathrm{We}$ investigate the feasibility of a 3D endoscopic transtubular interhemispheric transcallosal approach to the third ventricle.

\section{Methods}

Three-dimensional endoscopic interhemispheric transcallosal approaches with transseptal interforniceal, transforaminal, and transchoroidal modifications were performed through a tubular retractor system on 5 preserved cadaveric heads injected with colored latex - red for arteries and blue for veins (Fig. 1). A total of 15 procedures were performed, with each modification performed 5 times. Dissections were completed using $0^{\circ}$ and $45^{\circ} 2 \mathrm{D}$ optics (4-mm diameter, 18-cm length; Karl Storz Endoscopy-America, Inc.) and $0^{\circ} 3 \mathrm{D}$ optics (VSIII; Visionsense, Ltd.). Images were recorded and stored using the Karl Storz AIDA system and the Visionsense software, respectively. An Anspach eMax 2 Plus (Synthes, Inc.) electric neurosurgical drill was used.

\section{Neuronavigation}

For image-guided neuronavigation, 6 skin markers (fiducials) that appear on CT scans were affixed to the cranium. One-millimeter spiral CT axial slices were obtained (Biograph TruePoint PET•CT, Siemens AG) and transferred to the neuronavigation workstation (Kolibri Image-Guided Surgery Platform, Brainlab AG) for spatial registration.

\section{Transcallosal Approach}

Three-point fixation was achieved with the head positioned supine with $30^{\circ}$ neck flexion. Registration with the neuronavigation was completed and the software was used to identify the ideal entry point and trajectory to the third ventricle (Fig. 2). The boundaries of the superior sagittal sinus were identified and digitally marked. Following a linear incision, a $1.5-$ to $2-\mathrm{cm}$ midline parasagittal bur hole was fashioned $1-2 \mathrm{~cm}$ lateral to the midline. A minicraniotomy was used on one specimen to compare it with the keyhole option.

The edge of the superior sagittal sinus was exposed and the dura was opened in a semicircular fashion and retracted medially over the sinus. The bridging veins were carefully exposed and preserved as much as possible. Initial dissection of the interhemispheric fissure was completed under 2D endoscopic magnification. The endoscope was then inserted into the interhemispheric fissure and a thorough arachnoid dissection was performed to facilitate safe insertion of the tubular retractor. The tubular retractor system $(12$-mm width $\times 8$-mm height $\times 70$-mm length, ViewSite Brain Access System [VBAS]; Vycor Medical,
Inc.) consisted of an introducer inside a working channel port (Fig. 3). The tubular retractor was mounted onto a self-retaining snake retractor (Mizuho America, Inc.) and advanced gently into the interhemispheric fissure (Fig. $4 \mathrm{~A})$.

The conical shape of the retractor allowed for safe and smooth insertion into the supracallosal space, and the peripheral anatomy was easily observed through the transparent walls of the retractor (Fig. 4B). The small opening at the tip of the introducer was used to dissect arachnoid obstructions to allow advancement with bayonetted microsurgical, endoscopic, and tube shaft instruments (Fig. 4C). Preservation of most of the bridging veins was accomplished. If separation of a bridging vein was necessary during advancement of the retractor, the introducer was temporarily removed to permit expanded access.

The cingulate gyrus, the $\mathrm{A}_{4}$ segment of the anterior cerebral artery and its pericallosal branches, and ultimately the corpus callosum were identified (Fig. 5A and B). The tip of the retractor was advanced just beyond the vascular structures toward the corpus callosum to provide visible retraction of the vessels (Fig. 5C). The pericallosal arteries were gently mobilized and moved to either one or both sides of the retractor's tip, depending on the surgeon's preference (Fig. 5A and B). The snake retractor was locked in place, the introducer was removed, and the endoscope-attached to a holding arm-was inserted into the tubular retractor. To avoid thermal injury, the endoscope was advanced to a point approximately $5 \mathrm{~mm}$ above the tip of the introducer. An approximately $1-\mathrm{cm}$ superficial corpus callosotomy was fashioned using bayonetted bipolar cautery and endoscopic microinstruments (Fig. 6). The introducer was then reinserted and the retractor was carefully advanced through the fenestration to provide progressive blunt dilation of the corpus callosum (Fig. 7 upper). The ependymal walls of the lateral ventricle and septum pellucidum were observed (Fig. 7 lower).

\section{Transforaminal Modification}

With the aid of neuronavigation, the retractor was angled slightly toward and inserted into the lateral ventricle of interest through the ependyma. The ventricular walls were explored using a $0^{\circ}$ angled endoscope and the thalamostriate vein, septal vein, foramen of Monro (FM), fornix, and choroid plexus were visualized with $30^{\circ}$ and $45^{\circ}$ endoscopic probes (Fig. 8). The tubular retractor was centered over the FM, and angled bayonetted microdissectors were inserted through the FM into the third ventricle (Fig. 9). Minimal superomedial retraction was applied to the fornix.

\section{Transchoroidal Modification}

The choroid plexus was mobilized and a 1-cm incision was fashioned starting from the FM, crossing the tenia fornicis, and extending to the subchoroidal fissure (Fig. 10 upper). Entry into the third ventricle was achieved lateral to the choroid plexus and caudal to the thalamostriate vein (Fig. 10 lower). The endoscope was advanced into the third ventricle, passing between the internal cerebral veins. Division of the thalamostriate vein was performed in 2 cases to facilitate retraction. 

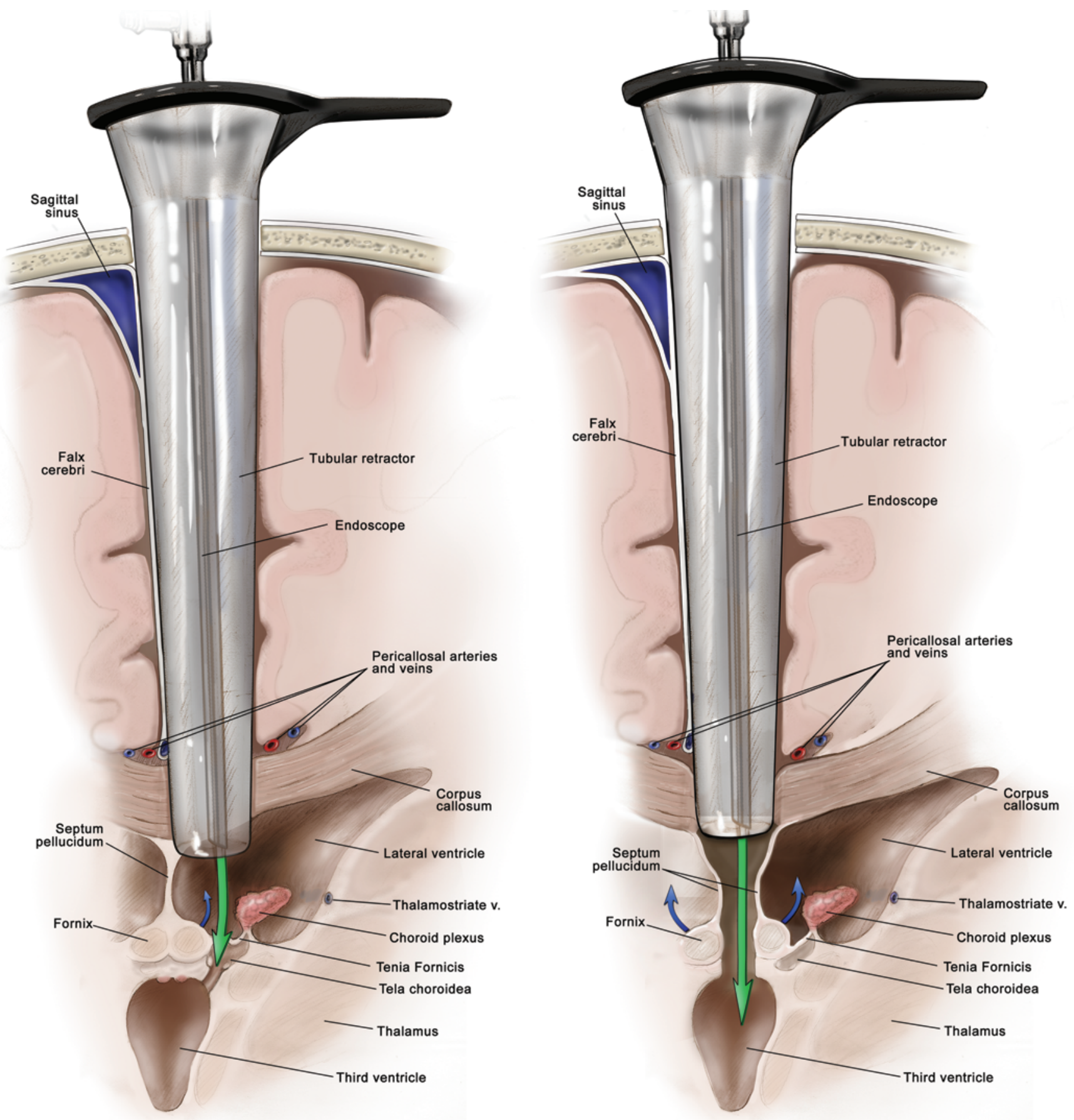

FIG. 1. Illustrations depicting the transforaminal, transchoroidal (left), and transseptal interforniceal (right) variations of the endoscopic transtubular transcallosal approach. The green arrows depict the direction of endoscopic visualization and dissection, and the blue arrows indicate the direction of retraction applied to the fornices. Copyright Antonio Bernardo. Published with permission.

\section{Transseptal Interforniceal Modification}

The 2 leaves of the septum pellucidum were separated at the midline by using blunt dissection, the raphe fornices were exposed and cut, and the fornices were separated by approximately $12 \mathrm{~mm}$ (Fig. 11 upper). ${ }^{30,38}$ The septal veins were not disturbed, minimal retraction was applied to the fornices, and the endoscope was advanced into the third ventricle between the forniceal columns (Fig. 11 lower). ${ }^{30}$

\section{Removal of the Retractor}

After removal of the retractor from the corpus callosum, the fenestration was visualized and inspected, and the pericallosal arteries were carefully explored for possible injury.

\section{Assessment of Exposure and Maneuverability}

The degree of exposure of the important surgical land- 


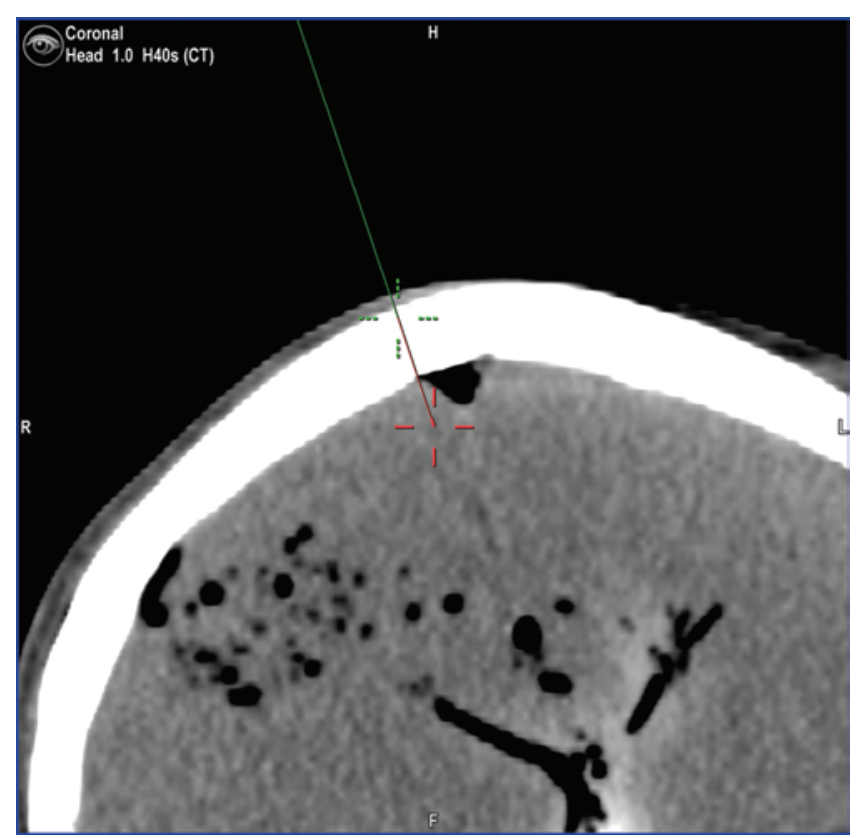

FIG. 2. A preoperative neuronavigation screenshot depicting identification of a safe entry zone that avoids the superior sagittal sinus.

marks was quantitatively evaluated and surgical maneuverability was qualitatively assessed for each approach and scored by 3 neurosurgeons (Tables 1 and 2). ${ }^{1,4,5}$ Exposure within the third ventricle was measured mediolaterally (between the medial walls of the thalamus) and anteroposteriorly (from the cerebral aqueduct to the infundibular
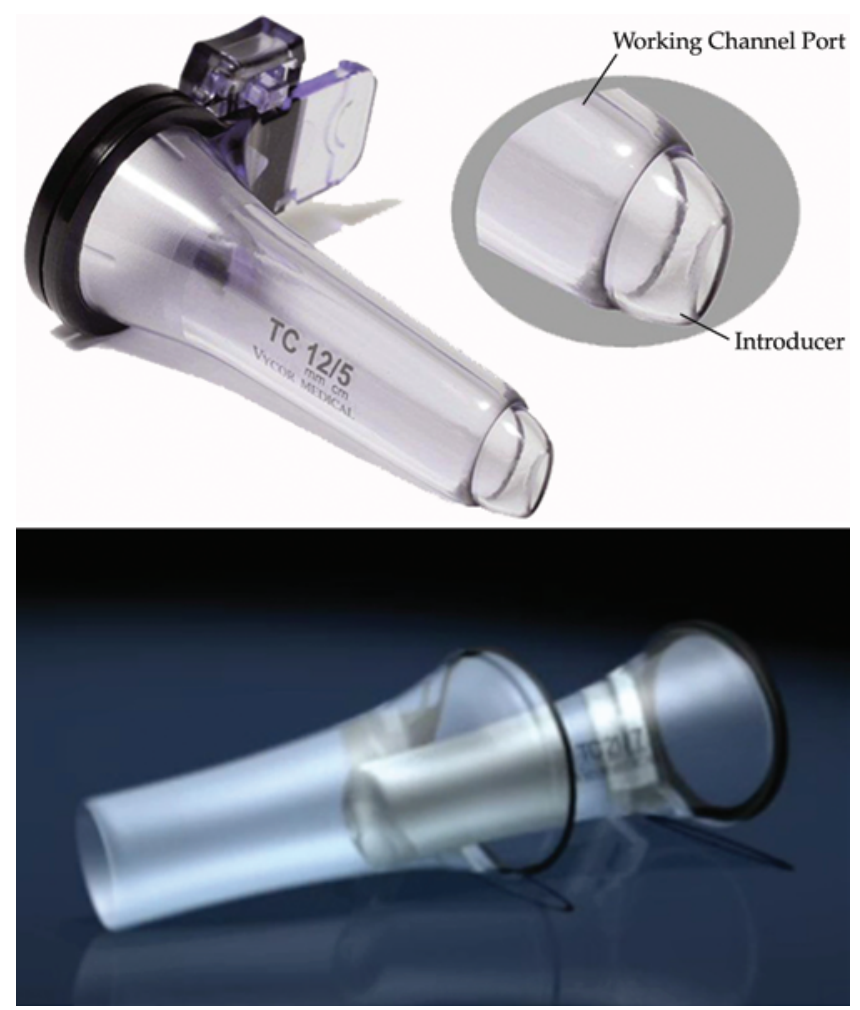

FIG. 3. Graphic depictions of the VBAS tubular retractor with the introducer in situ (upper) and with the introducer being removed (lower). recess) by using the neuronavigation pointer through the tubular retractor in each modification (Fig. 4A; Table 3). Surgical maneuverability was assessed to evaluate the possibility of performing bimanual surgical maneuvers on the exposed structures and within the third ventricle by using a combination of microinstruments, suction, and/or tube shaft instruments through the tubular retractor.

\section{Results \\ Tubular Retractor}

The conical shape of the retractor greatly facilitated its insertion and advancement, and its transparent walls provided excellent visualization of the peripheral anatomy-especially the pericallosal arteries. Small suction, microinstruments, and tube shaft instruments were all used through the retractor without difficulty. A neuronavigation pointer was also easily used through the retractor, and irrigation was applied through the retractor as needed without difficulty.

\section{Transforaminal Modification}

Excellent visualization of the lateral ventricles was achieved (Table 3), including visualization of the lateral walls, which are difficult to see microscopically. Key landmarks in the lateral ventricle were identified, including the choroid plexus, medial wall of thalamus, septal vein, and thalamostriate vein. The $30^{\circ}$ and $45^{\circ}$ angled endoscopes provided excellent visualization of the medial aspect of the lateral ventricle and of the FM. Angled dissectors were passed through the FM under direct endoscopic view and without difficulty. Neuronavigation was necessary in determining the optimal angle of insertion for the tubular retractor. An optimal trajectory into the lateral ventricle based on the specimen's specific anatomy was of paramount importance for achieving entry into the FM. All 3 surgeons scored this approach a "4" (multiangled exposure and facilitated surgical maneuverability), the maximum possible score; although surgical maneuverability was slightly limited around the floor of the third ventricle (Table 2). Because of its surgical route, this approach is optimal for accessing pathologies localized near the FM and for lesions that distend the body of the third ventricle and raise its roof.

\section{Transchoroidal Modification}

The choroid plexus, choroidal fimbria, and FM were identified after the retractor was inserted into the lateral ventricle. Following entry into the third ventricle and identification of the internal cerebral veins, the microinstruments were able to reach the contralateral side of the ventricle. The use of angled endoscopes was particularly beneficial in this approach, which also scored very high for exposure and surgical maneuverability except, as in other nonmidline approaches, for structures located at the floor of the third ventricle (Tables 2 and 3). Neuronavigation was extremely helpful for positioning and repositioning of the tubular retractor as needed during the procedure.

\section{Transseptal Interforniceal Modification}

Separation of the septal leaves was accomplished with 

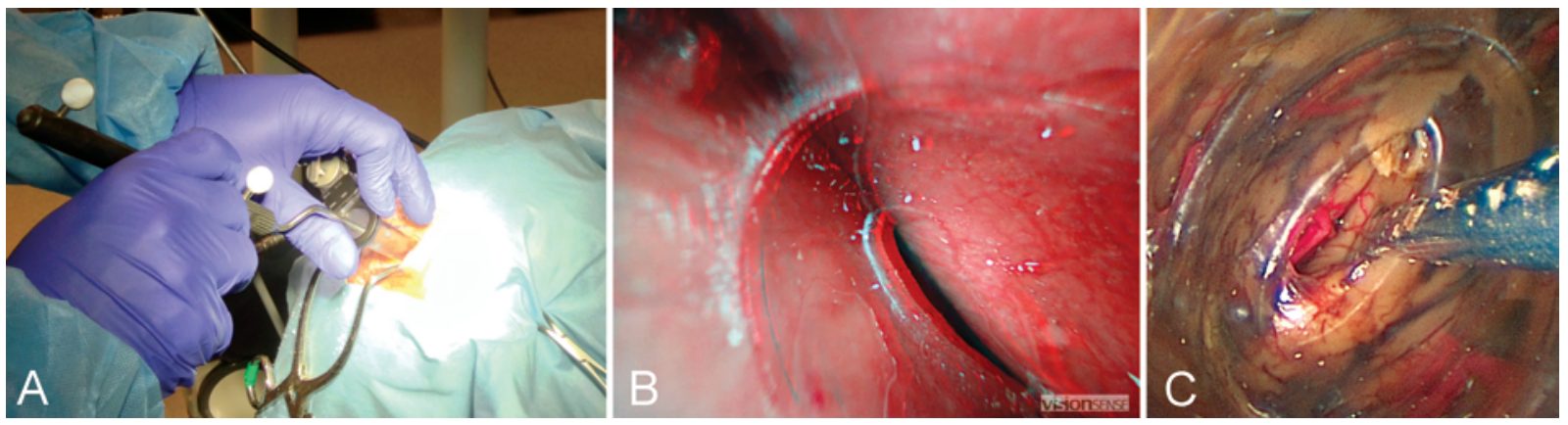

FIG. 4. Introduction and advancement of the tubular retractor into the surgical field. A: The tubular retractor, fastened to the selfretaining snake retractor, during insertion. B: View of the interhemispheric fissure through the tubular retractor's introducer. C: The opening in the tip of the introducer allowed for easy passage of microinstruments to release arachnoid adhesions.

minimal difficulty. After opening the roof of the third ventricle through the forniceal columns, the interthalamic adhesion-helpful for anatomical orientation and identification of the walls of the ventricle-was clearly visualized in all but one of the specimens. Accurate identification and bilateral displacement of the fornices was challenging, but was eased by use of the magnification provided by the endoscope. A curved dissector could be passed under the interthalamic adhesion without difficulty. Excellent visualization of the floor and lateral walls of the third ventricle was provided by a $0^{\circ}$ endoscope. Surgical maneuverability was facilitated within the third ventricle, and a $30^{\circ}$ endoscope enabled visualization of its posterior aspect. The $45^{\circ}$ endoscope allowed for exploration of the full length of the FM as well as visualization of the opening of the cerebral aqueduct and the suprapineal recess in all specimens (Tables 2 and 3). Because of its trajectory the interforniceal approach is not dependent on the size of the FM, and provides adequate access along a straight axis to the anterior portion and floor of the third ventricle. This approach is therefore most suitable for lesions primarily located in the inferior part of the third ventricle.

\section{Discussion}

Three categories of approaches have traditionally been used to access third ventricular lesions: endoscopic transcortical, microscopic transcortical, and transcallosal. ${ }^{9}$ Access to the third ventricle is virtually impossible without incising at least some neural structures. ${ }^{35}$ Determinants in selecting the best surgical route to a third ventricular lesion include lesion characteristics, location, pathology, size, relationship to adjacent structures, size of the lateral ventricles, and the surgeon's preferences. Other approaches, including the pterional, subfrontal, and subtemporal, are becoming less frequently used to access this area. Lesions affecting this part of the brain can be broadly categorized as intra- or extraaxial tumors (astrocytoma, ependymoma, colloid cyst, craniopharyngioma, and so on), congenital anomalies, and vascular malformations. ${ }^{9}$

The microscopic transcortical transventricular approach, which normally involves the opening or enlargement of the FM by opening the choroidal fissure, is traditionally used to approach lesions facing or protruding through the foramen. One of the main concerns with this approach is its risk of postoperative seizures, although several studies have contradicted previous findings of increased postoperative seizure activity. 7,15

Use of the endoscope in the transcortical approach has gained increasing popularity, ${ }^{6,12}$ although most surgeons prefer relatively large lateral ventricles when considering this type of approach. Some studies have also reported colloid cyst recurrence rates of up to $11.4 \%$ following endoscopic resection, most likely due to cyst residue in $62 \%$ of cases at the time of resection. ${ }^{12-22}$ Use of 3D endoscopy has helped by adding functional depth perception and facilitating anatomical understanding. ${ }^{4}$

The classic interhemispheric transcallosal approach
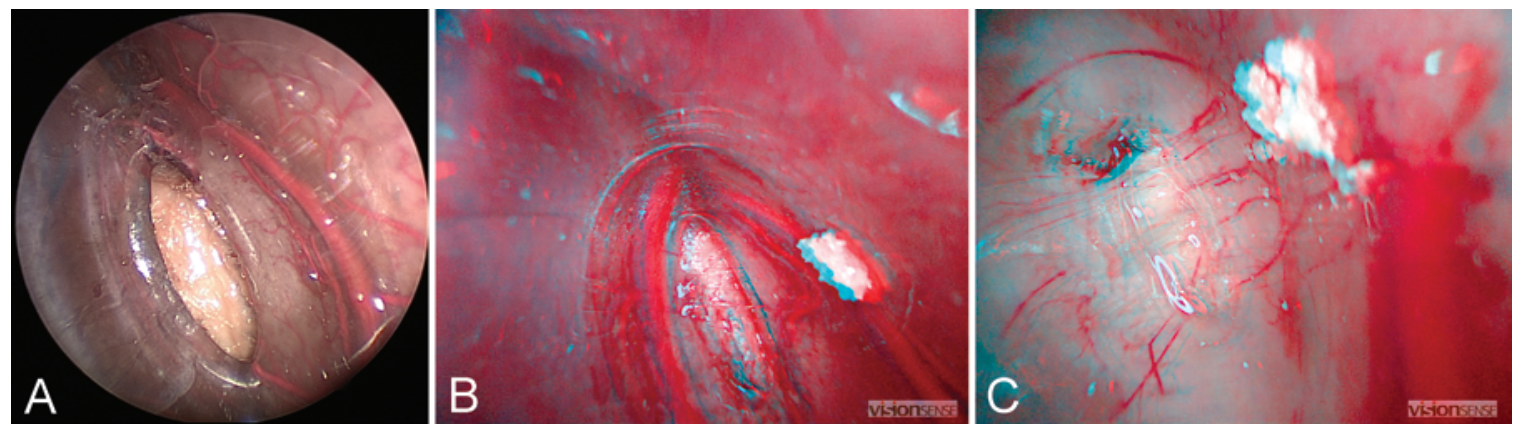

FIG. 5. Identification and retraction of the pericallosal arteries. A: A 2D endoscopic transtubular view of a unilateral pericallosal artery transposition. B and C: The 3D endoscopic transtubular views of a bilateral pericallosal artery retraction and the corpus callosum. The retractor facilitated safe retraction of the vessels around the tip of the device and provided adequate exposure of the corpus callosum. 

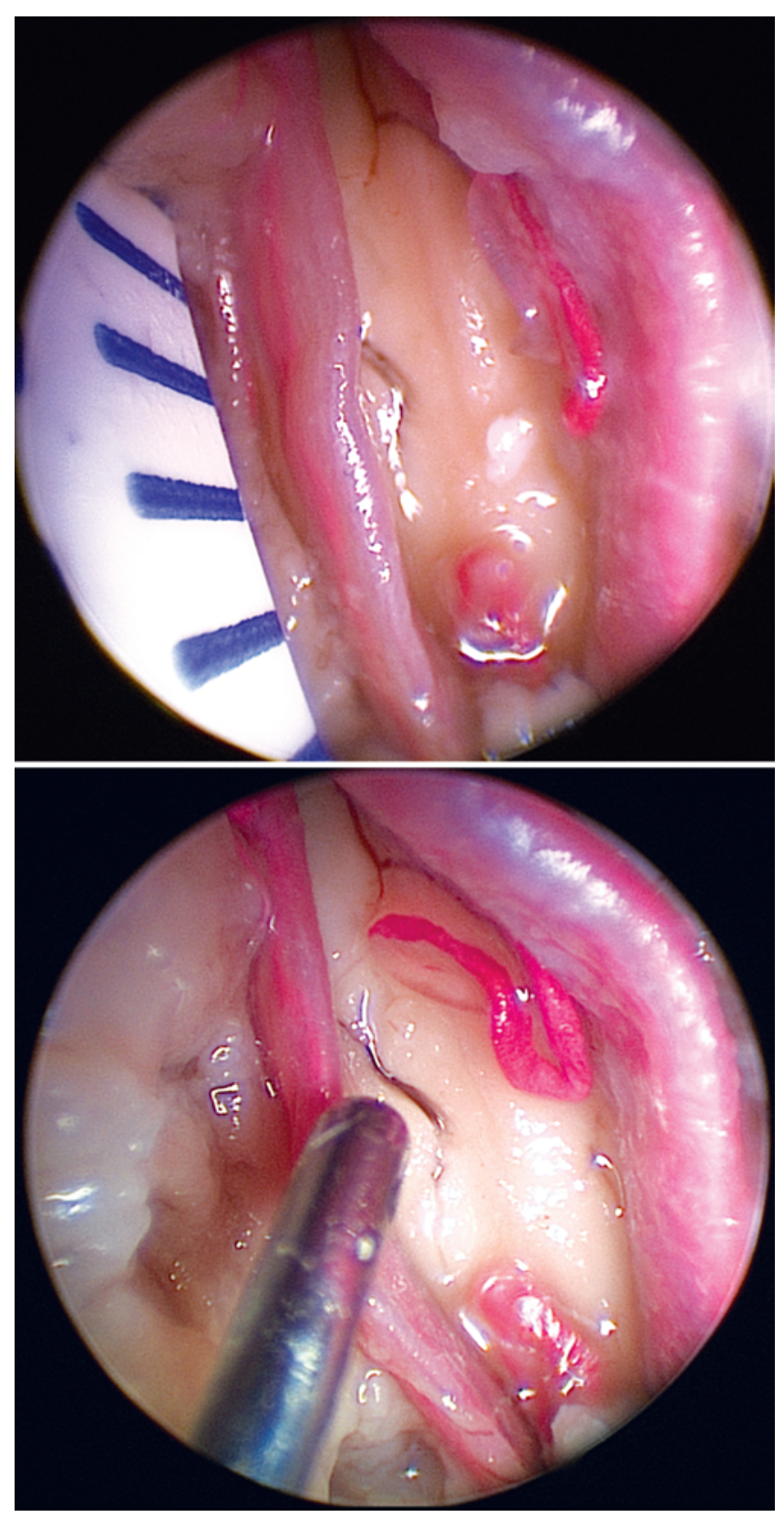

FIG. 6. Fenestration of the corpus callosum. Upper: A small opening in the corpus callosum was fashioned prior to advancement of the tubular retractor. Lower: The callosal arteries and a small feeding artery to the corpus callosum were visualized and safely retracted.

and its variants-transforaminal, transforaminal with unilateral incision of the column of the fornix, interforniceal, subchoroidal, and transchoroidal-are favorable options when ventriculomegaly is not a factor. ${ }^{6,24,37}$ For the purposes of this study, the transforaminal with unilateral incision of the fornix and subchoroidal variants were not included due to their rarity in clinical practice.

Although the transcallosal approach offers direct access to the third ventricle with minimal cortical resection, it also carries a risk of postoperative cognitive and memory deficits in the event of bilateral forniceal injury. Although disconnection syndrome has been attributed to
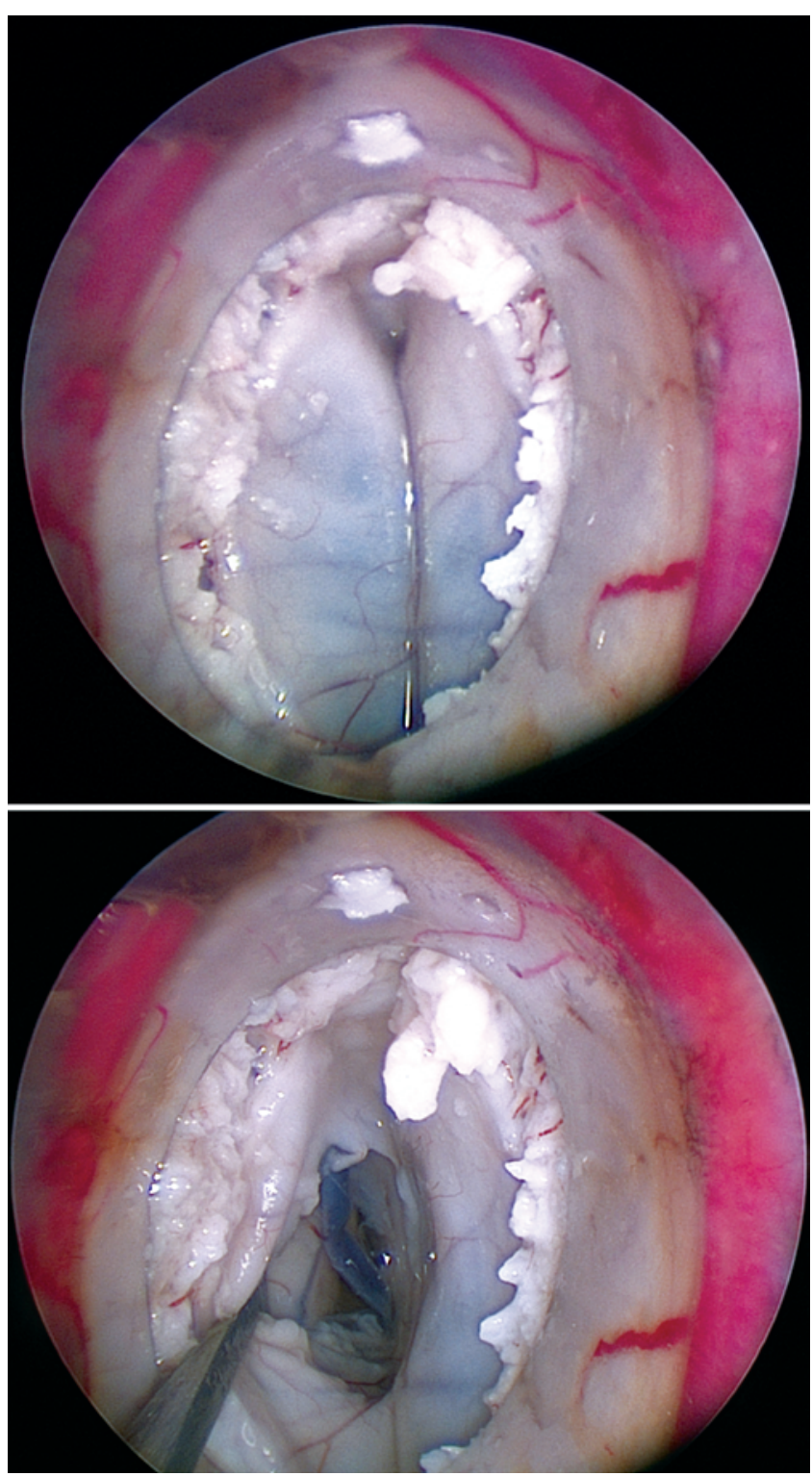

FIG. 7. Advancement of the tubular retractor through the callosotomy. Upper: Passage of the retractor tip through the fenestration in the corpus callosum. Lower: The ependymal lining of the lateral ventricles was observed.

callosotomy and transection of a large number of fibers, recent data support the role of the supracallosal gyrus in cognitive disturbances caused by the transcallosal approach. ${ }^{17}$ Peltier et al. conducted a detailed neurocognitive assessment in 8 patients who underwent a transcallosal approach, and showed higher incidences of dysexecutive syndrome and disturbances in interhemispheric transfer of learning in this small cohort of patients. ${ }^{25}$ As detailed in Mizowaki et al., a study published in 1982 by Apuzzo et al. reported a $33 \%$ risk of transient memory loss following this approach in a series of cases. ${ }^{24,36}$ Additionally, manipulation of the walls of the third ventricle could cause hypothalamic dysfunction and manifest as disturbances of consciousness, temperature control, respiration, and/or pituitary hormone status. ${ }^{28}$ 


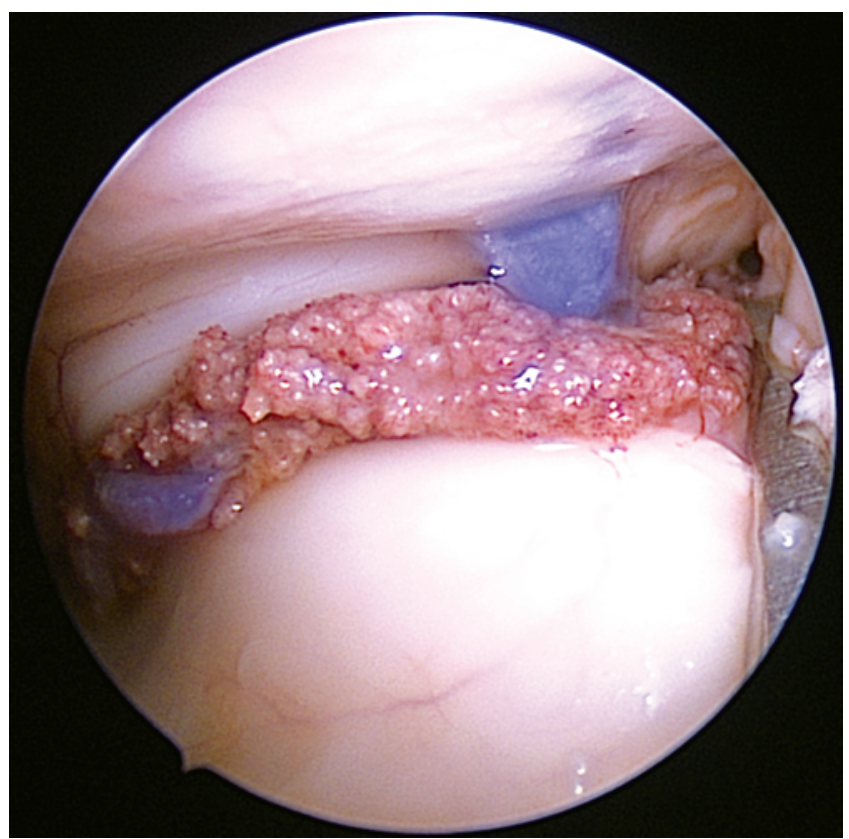

FIG. 8. The right lateral ventricle and choroid plexus seen through an angled 2D endoscope.

\section{Minimally Invasive Tubular Retractor Systems}

Since the introduction of retractors into neurosurgery by Greenberg in 1981, their use has been the subject of much debate. ${ }^{13,14,31,39}$ There have been several reports on the potentially deleterious effects of retractors on cortical microcirculation and resultant neural damage. Rosenørn and Diemer found an increased incidence of cortical damage from retractors held in place for more than 15 minutes with $20 \mathrm{~mm} \mathrm{Hg}$ of pressure in a rat model..$^{31}$ Additionally, systemic intraoperative factors, such as hypotension and blood loss, can increase vulnerability to retractor-mediated cortical ischemia. ${ }^{27}$ Whereas some authors have advocated the benefits of retractorless surgery, others have sought an alternative that provides surgical maneuverability while minimizing its adverse effects. ${ }^{21}$

In 1988, Kelly et al. first used a tubular retractor system mounted to a stereotactic frame for intraparenchymal tumor debulking. ${ }^{20}$ Since then, the use of frameless stereotactic techniques combined with minimally invasive tubular retractor systems has shown evidence of faster recovery times and lower morbidity rates. ${ }^{16}$ In recent years there have been several reports on cranial applications of tubular retractors. In 2008, Harris et al. reported on the successful use of "ventriculoport," a modified thoracic port with a diameter of $11.5 \mathrm{~cm}$, as a conduit for endoscope-assisted intraparenchymal tumor resection. ${ }^{16}$ In 2008, Dorman used modified spinal sequential dilators to resect 2 intraaxial tumors. ${ }^{10}$ Additional studies have successfully examined stereotactic transcortical retractor-guided resection of colloid cysts and other intraventricular lesions..$^{3,32}$

The use of tubular retractors in cranial surgery has been preliminarily shown to reduce pressure on cortical surfaces, minimize damage to white matter tracts, and potentially reduce intraoperative injury caused by surgical instruments. The retractor exerts reduced (compared with
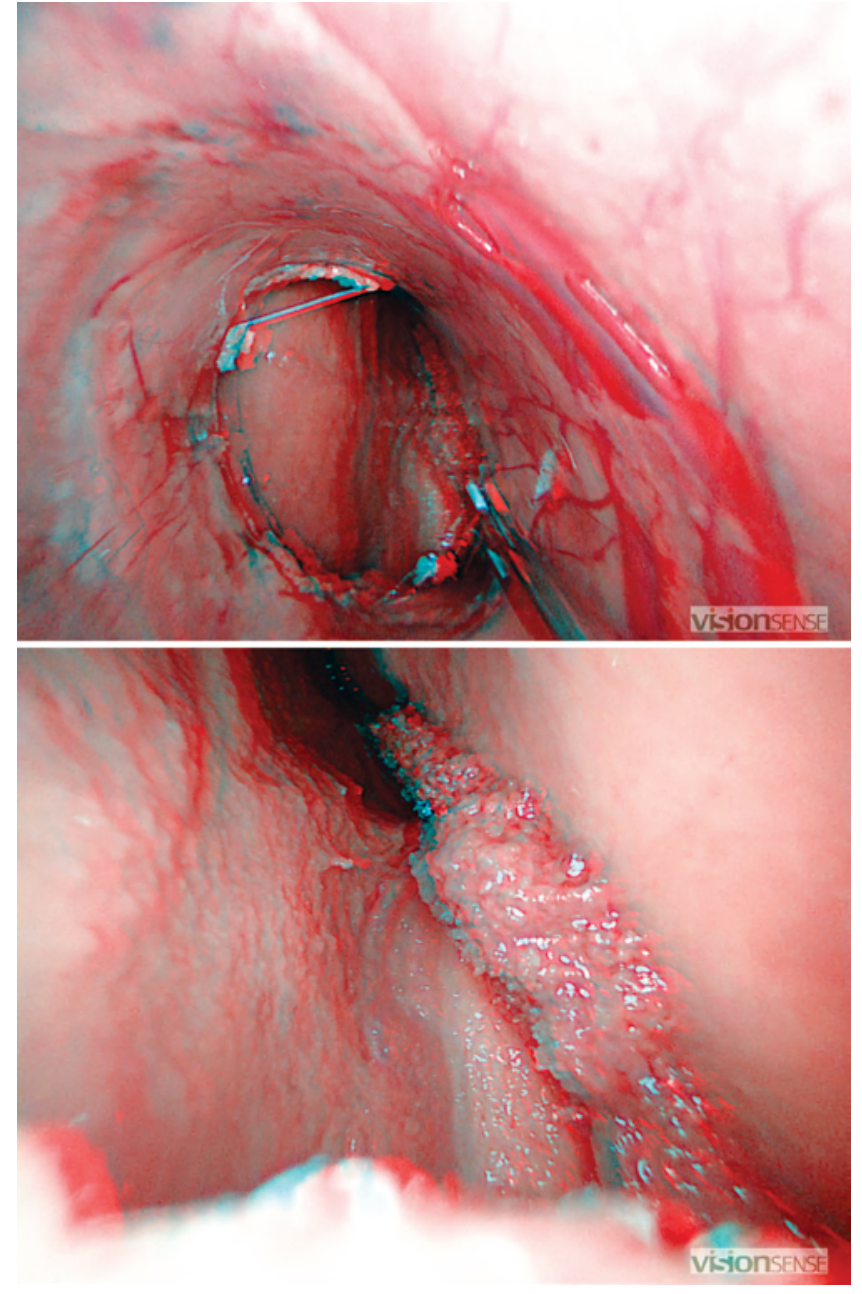

FIG. 9. Transforaminal modification. The FM was observed through the tubular retractor (upper) and the endoscope was advanced to visualize the choroid plexus (lower).

brain spatulas) and symmetrically distributed pressure as low as less than $10 \mathrm{~mm} \mathrm{Hg}$ onto impacted surface areas, reducing the risk of neuronal damage. ${ }^{27}$ Additionally, the blunt tip of the retractor is designed to provide progressive dilation that minimizes retraction injury to white matter tracts. ${ }^{14}$

Postoperative MRI data, obtained following use of the tubular retractor, have shown minimal T2/FLAIR changes along the surgical corridor, indicating minimal tissue damage and ischemia. ${ }^{14,16,26}$ Furthermore, by limiting the range of instrument movement and protecting the surrounding tissue from instruments within the working channel, the tubular retractor may reduce inadvertent injury to tissues during insertion or removal of instruments.

In this study, the VBAS provided a transparent structure that allowed for visual hemostatic monitoring of the retracted cortex throughout the procedure. The VBAS, a commercially available retractor system, has been previously used in transcortical approaches. ${ }^{11,18,27}$ In comparison with transcortical endoscopic surgery, our technique facilitates controlled removal of ventricular CSF by suction and formation of an air medium that provides better 

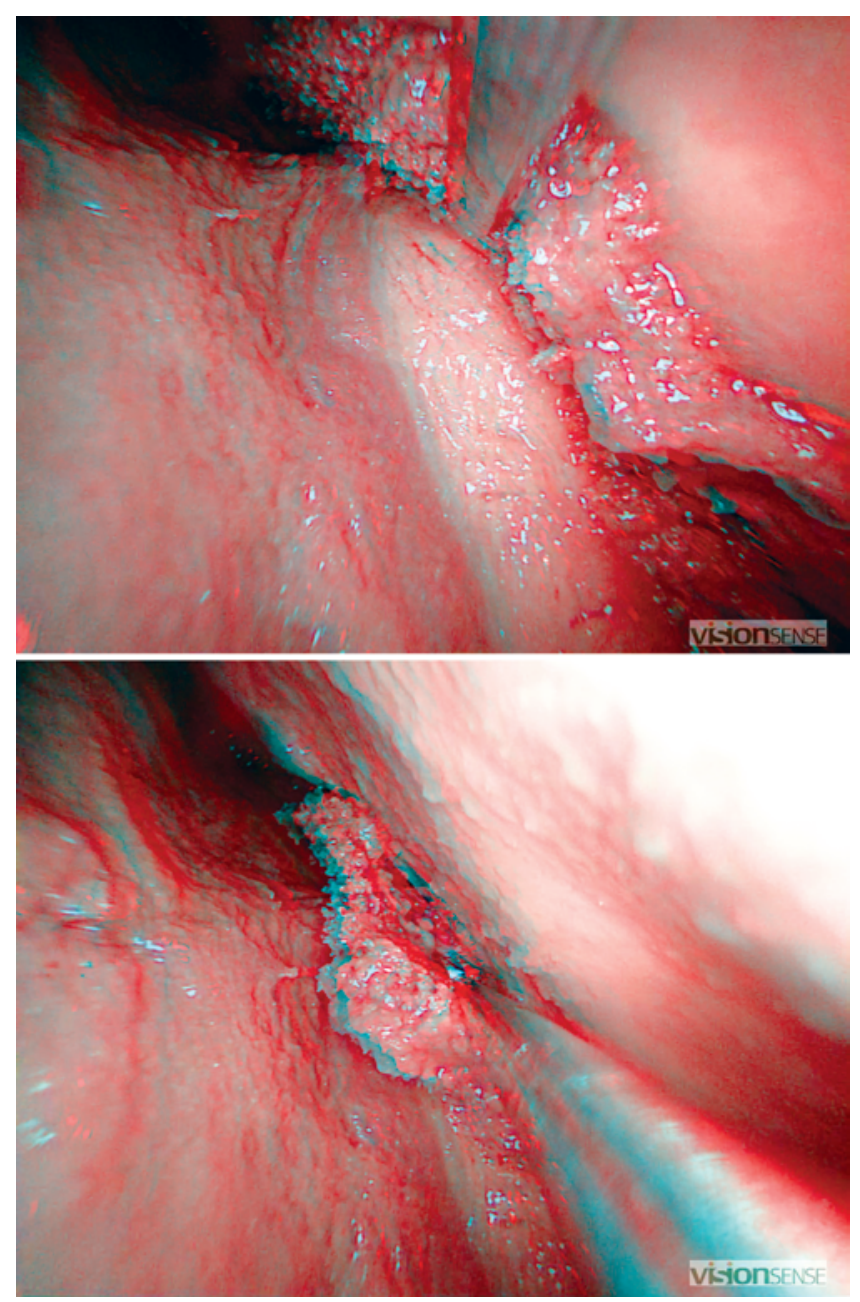

FIG. 10. Transchoroidal modification. The subchoroidal fissure was located (upper) and the transchoroidal modification was performed (lower).

intraoperative visualization. ${ }^{16,19}$ We hypothesize that the use of this retractor may, in addition to the aforementioned benefits, reduce the risk of thermal injury to surrounding tissues caused by the endoscope light or electrocautery. Furthermore, when compared with freehand manipulation of the retractor, we believe that use of the a self-retaining snake retractor may also decrease the risk of parietal cortex injury from torque effect. ${ }^{26}$

The $12 \mathrm{~mm}$ width $\times 8 \mathrm{~mm}$ height $\times 70 \mathrm{~mm}$ length VBAS was found to be most suitable for this study. There was sufficient space within this retractor to allow for simultaneous placement of the endoscope and a small suction aspirator, microinstrument, or tube shaft instrument. Instruments could be interchanged easily and quickly-with the surrounding tissues protected from accidental injury. If the use of a larger instrument or device is necessary, a retractor with a larger distal port size may be used. Tube shaft instruments and long endoscopic instruments were most efficient geometrically and ergonomically, and provided more space for movement within the retractor. Development of instruments specifically designed to accompany this system could contribute to the safety of this technique.

Future designs of this system, specifically for the trans-
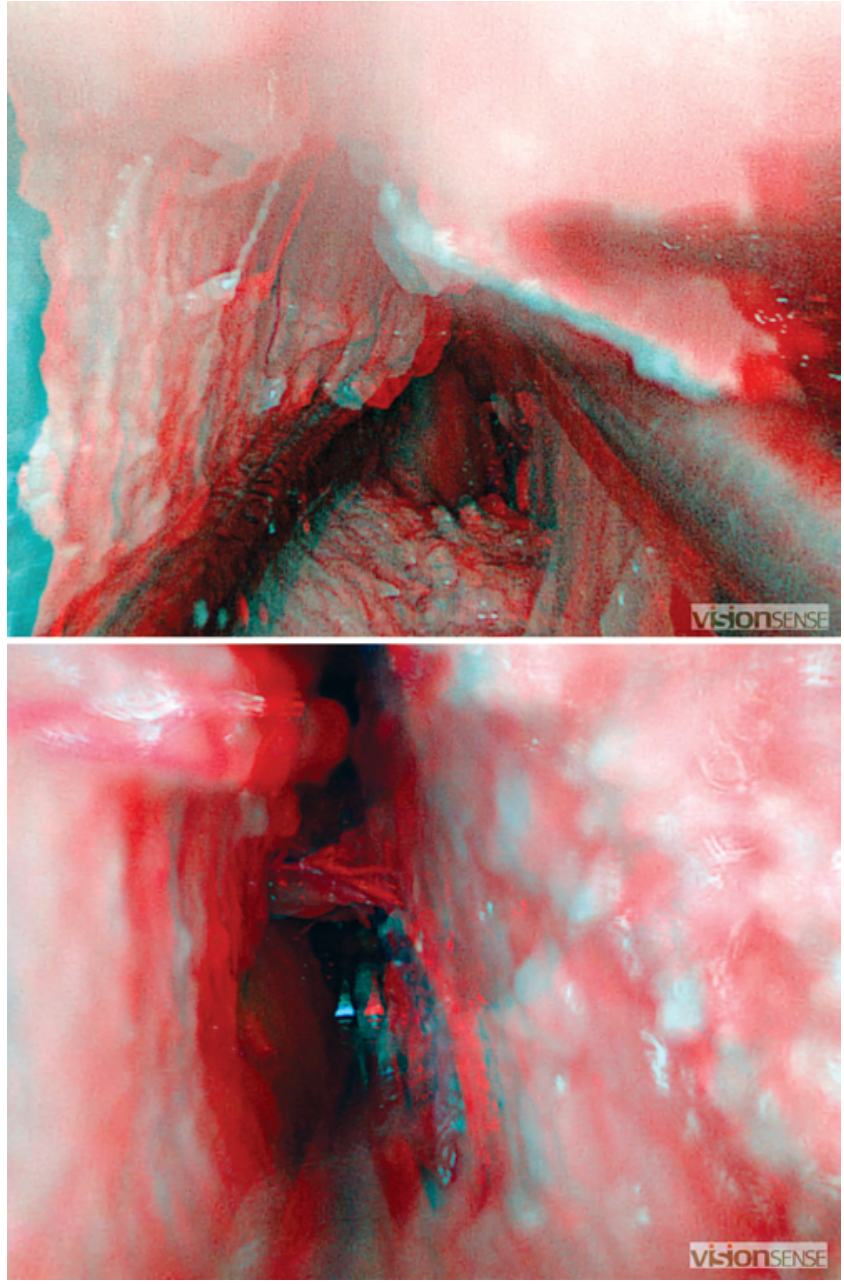

FIG. 11. Transseptal interforniceal modification. The leaflets of the septum (upper) and the fornices (lower) were divided to enable access into the third ventricle.

callosal approaches, may benefit from additional lengths, diameters, and shapes. A separate port for the endoscope within the retractor could be advantageous, but risks restricting the endoscope's range of movement. Although a neuronavigation pointer was used without difficulty here, a retractor with integrated neuronavigation markers would greatly ease insertion and enable constant monitoring of position and trajectory relative to anatomical landmarks.

Use of the 3D endoscope provided valuable stereoscopic perception, but surgeons should be familiar with the associated transtubular anatomy, as well as the use of

\section{TABLE 1. Exposure score definitions*}

\begin{tabular}{cl}
\hline Score & \multicolumn{1}{c}{ Exposure } \\
\hline 0 & No exposure \\
\hline 1 & Limited exposure; surgical maneuvers are not possible \\
\hline 2 & Multiangled exposure; surgical maneuvers are difficult \\
\hline 3 & Limited exposure; surgical maneuvers are possible \\
\hline 4 & Multiangled exposure; surgical maneuvers are facilitated \\
\hline
\end{tabular}

\footnotetext{
* Based on Bernardo et al., 2013.
} 
TABLE 2. Mean surgical exposure and maneuverability scores*

\begin{tabular}{lccc}
\hline \multirow{2}{*}{ Anatomical Structure } & \multicolumn{3}{c}{ Approach Variant \& Score } \\
\cline { 2 - 4 } Choroid plexus of third ventricle & Transforaminal & Transchoroidal & Transseptal Interforniceal \\
\hline Medial wall of thalamus & 4 & 4 & 4 \\
\hline Infundibular recess & 4 (contralat)/3 (ipsilat) & 4 (contralat)/3 (ipsilat) & 4 (bilat) \\
\hline Mammillary bodies & 3 & 3 & 4 \\
\hline Foramen of Monro & 3 & 3 & 4 \\
\hline Tela choroidea & 4 & 4 & 4 \\
\hline Tuber cinereum & 4 & 4 & 4 \\
\hline Cerebral aqueduct & 3 & 3 & 4 \\
\hline Forniceal columns & 4 & 4 & 4 \\
\hline Interthalamic adhesion & 4 & 4 & 4 \\
\hline
\end{tabular}

* The $30^{\circ}$ or $45^{\circ}$ angle endoscopes were used for visualization of structures via the transforaminal and transchoroidal approaches.

instruments through a tubular retractor, before attempting a transtubular approach. Although the retractor provides a safe corridor for the approach, it should not provide the surgeon with a false sense of safety-awareness of the position and anatomical surroundings of each instrument is still paramount to prevent tissue damage. Minor changes in the angle of the retractor were easily achieved without reinsertion, but any change in the retractor's trajectory should be made with great care to avoid damaging the cingulate gyrus. Using this retractor system in cases of large third ventricular tumors where mass effect significantly distorts the anatomy can be very challenging. Each surgeon who participated in this study spent several hours practicing using different instruments to handle objects such as small paper cubes and tissue paper through the retractor, to gain familiarity with transtubular technique before attempting it on cadavers. Despite the learning curve associated with transtubular techniques, the principles are mainly the same as those of microsurgery. ${ }^{26}$

\section{Conclusions}

The endoscopic transtubular transcallosal approach is a valid and potentially safer option for the management of third ventricular lesions. The tubular retractor provides rigid, constant, and symmetrically distributed pressure on the corpus callosum, and allows for progressive blunt dilation of the corpus callosum that may minimize retraction injury. Furthermore, this technique provides an added degree of safety by limiting the free range of instrumental

TABLE 3. Mean measurements of surgical exposure

\begin{tabular}{lcc}
\hline \multirow{2}{*}{ Approach Variant } & \multicolumn{2}{c}{ Length $(\mathrm{mm})$} \\
\cline { 2 - 3 } & Anteroposterior* & Mediolat $\dagger$ \\
\hline Transforaminal $\neq$ & $26.7 \pm 0.3$ & $3.2 \pm 0.4$ \\
\hline Transchoroidal & $26.9 \pm 0.6$ & $3.1 \pm 0.3$ \\
\hline Transseptal interforniceal & $27.2 \pm 0.5$ & $3.1 \pm 0.4$ \\
\hline
\end{tabular}

* Measured from the cerebral aqueduct posteriorly to the infundibular recess anteriorly.

$\dagger$ Measured between the medial walls of the thalamus.

$\ddagger$ With the use of $30^{\circ}$ or $45^{\circ}$ angle endoscopes. movement. The combination of 3D endoscopic visualization and a clear plastic retractor facilitates safe direct monitoring of the corridor during and after insertion.

\section{References}

1. Ammirati M, Bernardo A: Analytical evaluation of complex anterior approaches to the cranial base: an anatomic study. Neurosurgery 43:1398-1408, 1998

2. Bahuleyan B, Vogel TW, Robinson S, Cohen AR: Endoscopic total corpus callosotomy: cadaveric demonstration of a new approach. Pediatr Neurosurg 47:455-460, 2011

3. Barlas O, Karadereler S: Stereotactically guided microsurgical removal of colloid cysts. Acta Neurochir (Wien) 146:1199-1204, 2004

4. Bernardo A, Boeris D, Evins AI, Anichini G, Stieg PE: A combined dual-port endoscope-assisted pre- and retrosigmoid approach to the cerebellopontine angle: an extensive anatomo-surgical study. Neurosurg Rev 37:597-608, 2014

5. Bernardo A, Evins AI, Visca A, Stieg PE: The intracranial facial nerve as seen through different surgical windows: an extensive anatomosurgical study. Neurosurgery 72 (2 Suppl Operative):ons194-ons207, 2013

6. Cappabianca P, Cinalli G, Gangemi M, Brunori A, Cavallo LM, de Divitiis E, et al: Application of neuroendoscopy to intraventricular lesions. Neurosurgery 62 (2 Suppl 2):575598, 2008

7. Cohen-Gadol AA: Minitubular transcortical microsurgical approach for gross total resection of third ventricular colloid cysts: technique and assessment. World Neurosurg 79:207. e7-207.e10, 2013

8. Cohen-Gadol AA, Geryk B, Binder DK, Tubbs RS: Conquering the third ventricular chamber. J Neurosurg 111:590-599, 2009

9. Danaila L, Radoi M: Surgery of tumors of the third ventricle region. Chirurgia (Bucur) 108:456-462, 2013

10. Dorman JK: Tumor resection utilizing a minimally invasive spinal retractor with a novel cranial adaptor. Minim Invasive Neurosurg 51:358-360, 2008

11. Engh JA, Lunsford LD, Amin DV, Ochalski PG, Fernandez-Miranda J, Prevedello DM, et al: Stereotactically guided endoscopic port surgery for intraventricular tumor and colloid cyst resection. Neurosurgery 67 (3 Suppl Operative):ons198-ons205, 2010

12. Garrett M, Consiglieri G, Nakaji P: Transcranial minimally invasive neurosurgery for tumors. Neurosurg Clin N Am 21:595-605, v, 2010 
13. Greenberg IM: Self-retaining retractor and handrest system for neurosurgery. Neurosurgery 8:205-208, 1981

14. Greenfield JP, Cobb WS, Tsouris AJ, Schwartz TH: Stereotactic minimally invasive tubular retractor system for deep brain lesions. Neurosurgery 63 (4 Suppl 2):334-340, 2008

15. Guerrero MH, Cohen AR: Endoscope-assisted microsurgery of the corpus callosum. Minim Invasive Neurosurg 46:5456,2003

16. Harris AE, Hadjipanayis CG, Lunsford LD, Lunsford AK, Kassam AB: Microsurgical removal of intraventricular lesions using endoscopic visualization and stereotactic guidance. Neurosurgery 62 (2 Suppl 2):622-629, 2008

17. Harvey AS, Rosenfeld JV: What happens to cognitive function following surgery for hypothalamic hamartoma? Neurology 81:1028-1029, 2013

18. Herrera SR, Shin JH, Chan M, Kouloumberis P, Goellner E, Slavin KV: Use of transparent plastic tubular retractor in surgery for deep brain lesions: a case series. Surg Technol Int 19:47-50, 2010

19. Jho HD, Alfieri A: Endoscopic removal of third ventricular tumors: a technical note. Minim Invasive Neurosurg 45:114-119, 2002

20. Kelly PJ, Goerss SJ, Kall BA: The stereotaxic retractor in computer-assisted stereotaxic microsurgery. Technical note. J Neurosurg 69:301-306, 1988

21. Lanzino G: Editorial. Retractorless brain surgery. J Neurosurg 116:290, 2012

22. Longatti P, Godano U, Gangemi M, Delitala A, Morace E, Genitori L, et al: Cooperative study by the Italian neuroendoscopy group on the treatment of 61 colloid cysts. Childs Nerv Syst 22:1263-1267, 2006

23. Milligan BD, Meyer FB: Morbidity of transcallosal and transcortical approaches to lesions in and around the lateral and third ventricles: a single-institution experience. Neurosurgery 67:1483-1496, 2010

24. Mizowaki T, Nagashima T, Yamamoto K, Kawamura A, Yoshida M, Kohmura E: Optimized surgical approach to third ventriclular choroid plexus papillomas of young children based on anatomical variations. World Neurosurg [epub ahead of print

25. Peltier J, Roussel M, Gerard Y, Lassonde M, Deramond H, Le Gars D, et al: Functional consequences of a section of the anterior part of the body of the corpus callosum: evidence from an interhemispheric transcallosal approach. J Neurol 259:1860-1867, 2012 (Erratum in J Neurol 260:1199, 2013)

26. Raza SM, Recinos PF, Avendano J, Adams H, Jallo GI, Quinones-Hinojosa A: Minimally invasive trans-portal resection of deep intracranial lesions. Minim Invasive Neurosurg 54:5-11, 2011

27. Recinos PF, Raza SM, Jallo GI, Recinos VR: Use of a minimally invasive tubular retraction system for deep-seated tumors in pediatric patients. J Neurosurg Pediatr 7:516-521, 2011

28. Renn WH, Rhoton AL Jr: Microsurgical anatomy of the sellar region. J Neurosurg 43:288-298, 1975

29. Rosenfeld JV, Feiz-Erfan I: Hypothalamic hamartoma treatment: surgical resection with the transcallosal approach. Semin Pediatr Neurol 14:88-98, 2007

30. Rosenfeld JV, Freeman JL, Harvey AS: Operative technique: the anterior transcallosal transseptal interforniceal approach to the third ventricle and resection of hypothalamic hamartomas. J Clin Neurosci 11:738-744, 2004

31. Rosenørn J, Diemer N: The risk of cerebral damage during graded brain retractor pressure in the rat. J Neurosurg 63:608-611, 1985

32. Ross DA: A simple stereotactic retractor for use with the Leksell stereotactic system. Neurosurgery 32:475-476, 1993

33. Shiramizu H, Hori T, Matsuo S, Niimura K, Yoshimoto H, Ishida A, et al: Anterior callosal section is useful for the removal of large tumors invading the dorsal part of the anterior third ventricle: operative technique and results. Neurosurg Rev 36:467-475, 2013

34. Tebo CC, Evins AI, Christos PJ, Kwon J, Schwartz TH: Evolution of cranial epilepsy surgery complication rates: a 32-year systematic review and meta-analysis. J Neurosurg 120:1415-1427, 2014

35. Tomasello F, Cardali S, Angileri FF, Conti A: Transcallosal approach to third ventricle tumors: how I do it. Acta Neurochir (Wien) 155:1031-1034, 2013

36. Tubbs RS, Prekupec M, Loukas M, Hattab EM, Cohen-Gadol AA: The indusium griseum: anatomic study with potential application to callosotomy. Neurosurgery 73:312-316, 2013

37. Türe U, Yaşargil MG, Al-Mefty O: The transcallosal-transforaminal approach to the third ventricle with regard to the venous variations in this region. J Neurosurg 87:706-715, 1997

38. Winkler PA, Weis S, Büttner A, Raabe A, Amiridze N, Reulen HJ: The transcallosal interforniceal approach to the third ventricle: anatomic and microsurgical aspects. Neurosurgery 40:973-982, 1997

39. Yokoh A, Sugita K, Kobayashi S: Clinical study of brain retraction in different approaches and diseases. Acta Neurochir (Wien) 87:134-139, 1987

\section{Author Contributions}

Conception and design: Bernardo, Shoakazemi, Evins, Burrell. Acquisition of data: Bernardo, Shoakazemi, Evins, Burrell. Analysis and interpretation of data: Bernardo, Shoakazemi, Evins, Burrell. Drafting the article: Bernardo, Shoakazemi, Evins. Critically revising the article: Bernardo, Evins. Reviewed submitted version of manuscript: all authors. Approved the final version of the manuscript on behalf of all authors: Bernardo. Administrative/technical/material support: Evins, Burrell. Study supervision: Bernardo, Evins, Stieg.

\section{Supplemental Information \\ Previous Presentation}

Portions of this work were presented as proceedings at the 24th Annual Meeting of the North American Skull Base Society, held in San Diego, California, on February 15, 2014.

\section{Correspondence}

Antonio Bernardo, Department of Neurological Surgery, Weill Cornell Medical College, 525 E. 68th St., Box 99, New York, NY 10065. email: anb2029@med.cornell.edu. 